\title{
Statistical Approach to Estimated Uncertainty of Nuclear Concentration in Problems of Isotope Kinetics
}

\section{V. Kolesov, A. V. Novichkov, E. E. Voznyakevich, and A. M. Terekhova}

Obninsk Institute for Nuclear Power Engineering of the National Research Nuclear University MEPhl, Studgorodok 1, Obninsk, Kaluga region, 249040, Russia

\section{Abstract}

The minority of papers only is devoted to the study of impact of the uncertainties in nuclear data on the nuclear concentration received during the solution of the problem of fuel burn-up in the reactor facility. On the other hand, uncertainties of known reaction rates, neutron fluxes, etc. can lead to considerable distortions of the results obtained therefore it is important to be able to assess the impact of such uncertainties

Corresponding Author: V. V. Kolesov

VVKolesov@mephi.ru

Received: 23 December 2017

Accepted: 15 January 2018

Published: 21 February 2018

Publishing services provided by Knowledge $\mathrm{E}$

(c) V. V. Kolesov et al. This article is distributed under the terms of the Creative Commons

Attribution License, which permits unrestricted use and redistribution provided that the original author and source are credited.

Selection and Peer-review under the responsibility of the AtomFuture Conference Committee.

\section{G OPEN ACCESS} on nuclear concentration of nuclides. In this paper we consider the problem of the impact assessment of uncertainties in nuclear data on reactor functionalities as applied to isotope kinetics which represents the well-known Cauchy linear problem. The most exact approach is the statistical approach of the randomized selection of input parameters in using different distribution laws. But the simplest method of the analysis of sensitivity of model to perturbation parameters is the following (it has several names in the literature: one-at-a-time sensitivity measures, $1 \%$ sensitivity method): by varying one of the input parameters of the task for the small amount (for example, for $1 \%$ ) when other parameters are constant, the corresponding response of output parameters is defined (variation approach). Our results show that in burn-up calculations the mean square deviations of nuclear concentrations obtained using statistical approach coincide with the variations of nuclear concentrations obtained in the variation approach.

Keywords: reactor facility, burn-up calculations, statistical approach, variation approach, nuclear data uncertainties, nuclear concentration uncertainties.

\section{Introduction}

At the present day much attention is given to the assessments of the impact of uncertainties in nuclear data on parameters of various reactor facilities. However, in general the impact of uncertainties in nuclear data on standard functionalities ( $\mathrm{K}_{\text {eff, }}$ reaction rates etc.) obtained using sensitivity coefficients. The minority of papers only is 
devoted to the study of impact of the uncertainties in nuclear data on the nuclear concentration received during the solution of the problem of fuel burn-up in the reactor facility ([1-3]). The solution of fuel burn-up problems is important for assessment of accumulation of a number of isotopes arising in reactor facilities, such as, for example, of plutonium isotopes. At the same time in general it is rather difficult to determine experimentally their concentration in the spent fuel. Thus, numerical methods of solution of burn-out problems are of high importance now. On the other hand, uncertainties of known reaction rates, neutron fluxes, etc. can lead to considerable distortions of the results obtained therefore it is important to be able to assess the impact of such uncertainties on nuclear concentration of nuclides.

Propagation of uncertainties in nuclear data on the parameters which characterize the nuclear facility can be executed in a number of ways. At the present day the most known approach is an approach for obtaining the sensibility coefficients defined by the linear perturbation theory. In the end we obtain the linear equation system with respect to sensitivity coefficients.

The simplest method of the analysis of sensitivity of model to perturbation parameters is the following (it has several names in the literature: one-at-a-time sensitivity measures, $1 \%$ sensitivity method): by varying one of the input parameters of the task for the small amount (for example, for $1 \%$ ) when other parameters are constant, the corresponding response of output parameters (see e.g. [4]) is defined.

However, the most exact approach is the statistical approach of the randomized selection of parameters using different distribution laws. At the same time we can run both one and several parameters of the model considering them as independent parameters or with use of correlation between them.

Let us consider the problem of the impact assessment of uncertainties in nuclear data on reactor functionalities as applied to isotope kinetics which represents the wellknown Cauchy linear problem.

As per [5] let the differential equation system of the first order (in case of isotope kinetics - linear) be given for definition of nuclear concentration vector $\vec{N}(t, \vec{p})$ of $n$ dimension:

$$
\begin{gathered}
\frac{d \vec{N}(t, \vec{p})}{d t}=f(t, \vec{N}(t, \vec{p}), \vec{p})=K(\vec{p}) \vec{N}(t, \vec{p}) \\
\vec{N}(0, \vec{p})=\vec{N}_{0}(\vec{p}),
\end{gathered}
$$

where $K(\vec{p})$ - matrix of coefficients which depend on problem parameters (vector of parameters $\left.\vec{p}=\left(p_{1}, \ldots, p_{m}\right)\right)$, such as one-group neutron flux density, one-group reaction rates for different nuclides, etc. 
Perturbed solution $\vec{N}(t, \vec{p})+\Delta \vec{N}(t, \vec{p})$, which corresponds to the perturbed parameter column $\vec{p}_{i}+\Delta \vec{p}_{i}$ can be determined in the predicate of linear expansion (linear perturbation theory):

$$
N_{i}(t, \vec{p}+\Delta \vec{p}) \approx N_{i}(t, \vec{p})+\frac{\partial N_{i}(t, \vec{p})}{\partial p_{i}} \Delta p_{i}
$$

Whereby it is necessary to solve the problem with regards to the perturbations:

$$
\begin{gathered}
\frac{d \Delta \vec{N}(t, \vec{p})}{d t}=\frac{\partial \vec{f}(t, \vec{p})}{\partial \vec{N}} \Delta \vec{N}(t, \vec{p})+\frac{\partial \vec{f}(t, \vec{p})}{\partial \vec{p}} \Delta \vec{p} ; \\
\Delta \vec{N}(0, \vec{p})=0,
\end{gathered}
$$

Where elements of the matrix $\frac{\partial \vec{f}(t, \vec{p})}{\partial \vec{N}} \equiv\left[\frac{\partial f_{i}(t, \vec{p})}{\partial N_{k}}\right]$ of size: $n \times n$ and elements of the matrix $\frac{\partial \vec{f}(t, \vec{p})}{\partial \vec{p}} \equiv\left[\frac{\partial f_{i}(t, \vec{p})}{\partial p_{k}}\right]$ of size: $n \times m$, broadly speaking, depend on $t$ and, therefore they should be calculated on every step which involves a certain difficulty.

The so-called sensibility factors are often introduced; the matrix hereof is given by: $Z(t, \vec{p}) \equiv \frac{\partial \vec{N}(t, \vec{p})}{\partial \vec{p}} \equiv\left[\frac{\partial N_{i}(t, \vec{p})}{\partial p_{k}}\right]$ and it depends on $t$.

These factors can be estimated from the system $m \times n$ of linear equations:

$$
\begin{gathered}
\frac{d Z_{i k}(t, \vec{p})}{d t}=\frac{\partial \vec{f}_{i}(t, \vec{p})}{\partial \vec{N}_{k}} Z_{i k}(t, \vec{p})+\frac{\partial \vec{f}_{i}(t, \vec{p})}{\partial \vec{p}_{k}} ; \\
Z_{i k}(0, \vec{p})=0 .
\end{gathered}
$$

Application of the linear perturbation theory for problems of isotope kinetics are considered, for example, in [1] and [2].

In statistical approach let us consider that any of $p_{i}$ parameters is known with some uncertainty. Then $p_{i}$ is a random variable distributed under the lognormal law with the given mean value and variance (mean square deviation).

Let us study the impact of uncertainties in nuclear data on nuclear concentration of the nuclides obtained during solution of the problem of fuel burn-up, running the corresponding random variable with the given mean square deviation.

VisualBurnOut [6] was used as the program for calculation of fuel burn-up where, in turn, the one-group reaction rates and fluxes assumed in the special format which corresponded to the output format of the MCNP [7] program were used.

\section{Calculation results}

In order to study the impact of uncertainties of reaction rates on nuclear concentration let us consider the problem of fuel burn-up in the benchmark cell which represents a 
three-zone square cell (lattice spacing is $1.3127 \mathrm{~cm}$ ) with MOX fuel [8]. The structure of physical zones is given in Table 1.

TABLE 1: The structure of the cell used for burn-up calculations.

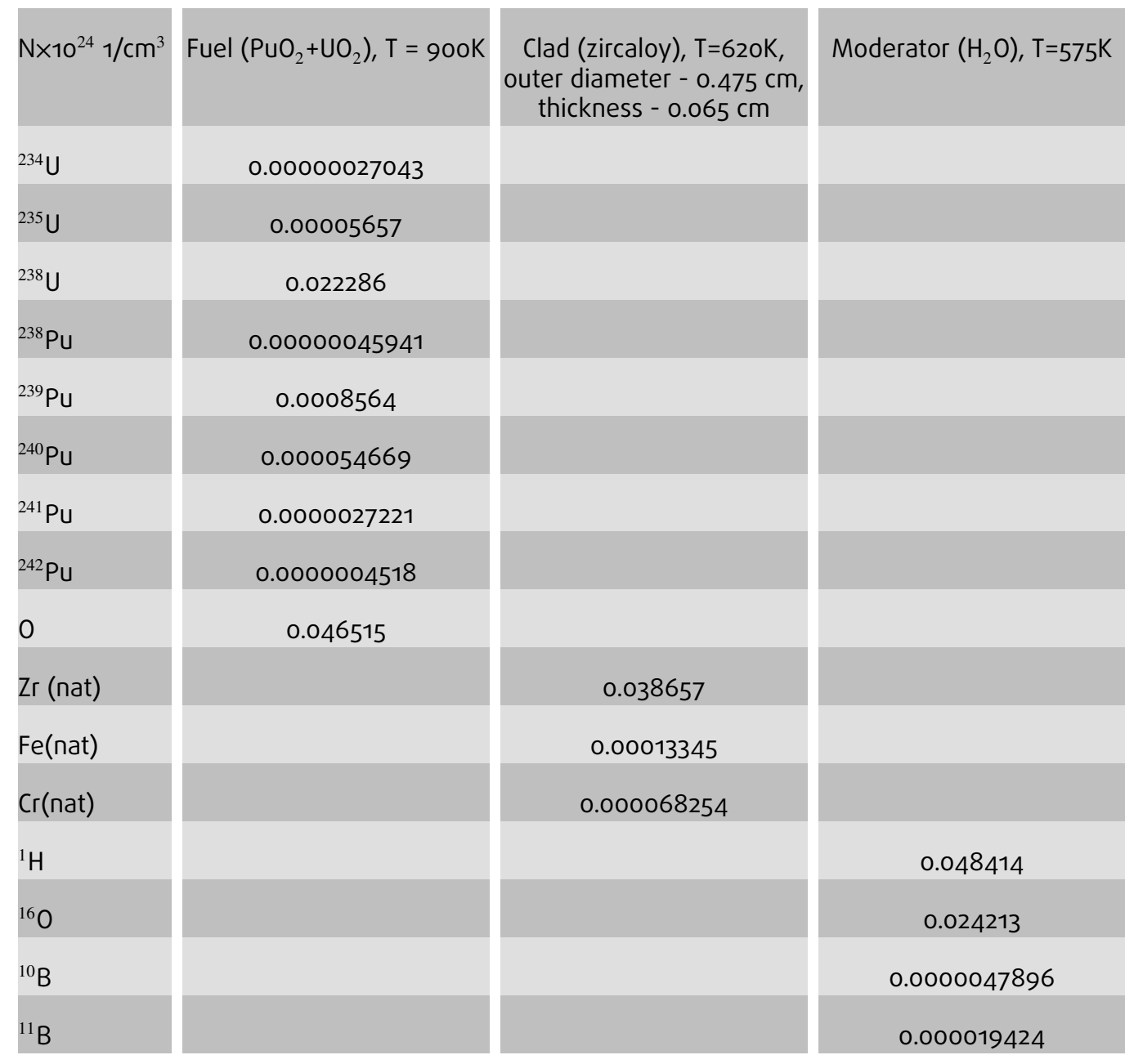

During execution of calculations of burn-up we assumed that mean square deviations of rates of fission and radiative capture of ${ }^{235} \mathrm{U}$ and ${ }^{239} \mathrm{Pu}$ made $10 \%$. According to the lognormal distribution law the corresponding reaction rate was repeatedly run, and every time the burn-up problem was solved. Burn-up calculations at perturbation of rates of fission and radiative capture were respectively performed for effective burnup periods of 100 and 500 days. The reaction rates were run 3000 times for obtaining the mean square deviations of nuclear concentration. In the course of calculations the neutron flux density assumed to be a constant.

As a result we obtained the random selection of nuclear concentration of nuclides. For example Figures 1 and 2 present the diagrams of distribution of nuclear concentrations ${ }^{90} \mathrm{Y}$ (for 100 days) and ${ }^{238} \mathrm{Pu}$ (for 500 days). 


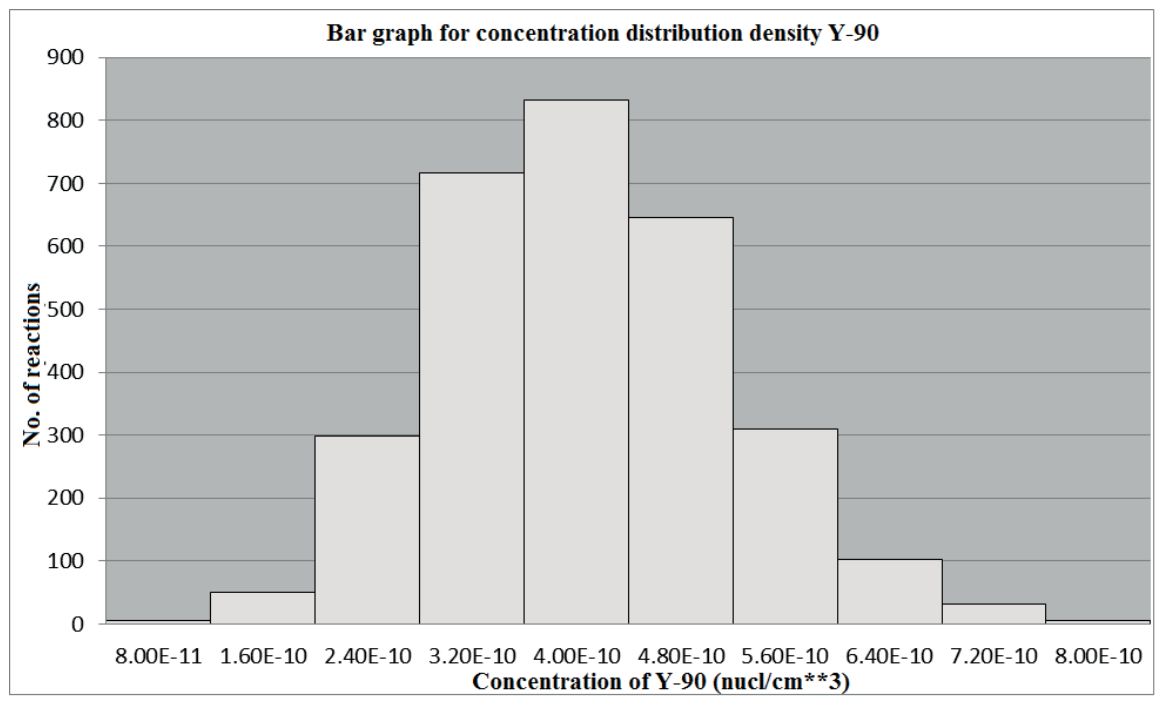

Figure 1: Distribution of nuclear concentration ${ }^{90} \mathrm{Y}$ at $10 \%$ perturbation of ${ }^{235} \mathrm{U}$ and ${ }^{239} \mathrm{Pu}$ fission rate at burn-up within 100 days and neutron flux density $\mathrm{F}=3 \cdot 10^{14} \mathrm{~cm}^{-2} \mathrm{~s}^{-1}$.

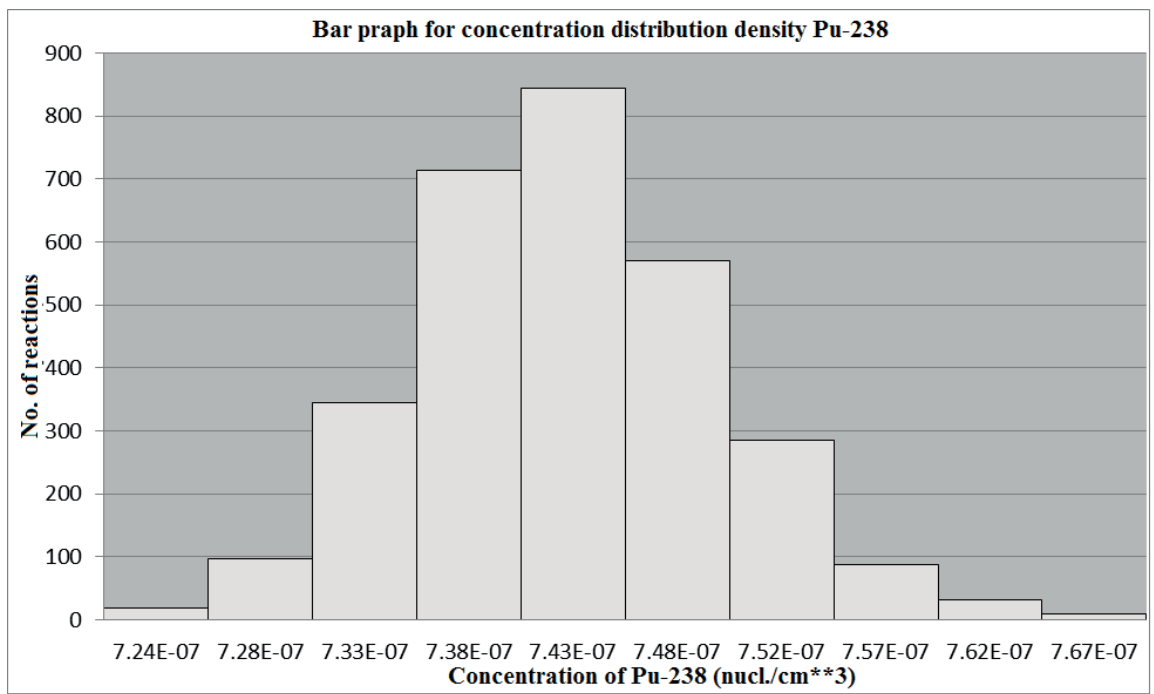

Figure 2: Distribution of nuclear concentration ${ }^{238} \mathrm{Pu}$ at $10 \%$ perturbation of ${ }^{235} \mathrm{U}$ and ${ }^{239} \mathrm{Pu}$ radiative capture rate at burn-up within 100 days and neutron flux density $\mathrm{F}=3 \cdot 10^{14} \mathrm{~cm}^{-2} \mathrm{~s}^{-1}$.

The results obtained by us for mean square deviations of nuclear concentration were compared to the results obtained by means of the so-called one-at-a-time sensitivity measures (hereinafter referred to as variation approach). The main point of these approaches as already mentioned is the variation of any input parameter and study of the impact of this variation for the output parameters. In this approach the variation of the rates made also $10 \%$ and we studied the obtained variations in nuclear concentrations. 
If several reaction rates varied, the calculation of the full variation was performed as follows:

$$
\text { totalvar }=\sqrt{\sum_{i=1}^{n} \operatorname{var}_{i}^{2}}
$$

The result obtained by us for both approaches are given in tables 2 and 3 .

TABLE 2: Comparison of statistical and variation approach for assessment of uncertainties of nuclear concentration of ${ }^{90} \mathrm{Y}$.

\begin{tabular}{c}
$\begin{array}{c}\text { Statistical approach (mean square deviations } \\
\text { of the run reaction rate }-10 \% \text {, burn-out } \\
\text { period - } 100 \text { days) }\end{array}$ \\
$\begin{array}{c}\text { Run parameter } \\
\begin{array}{c}\text { Nuclear concentration } \\
\text { of }{ }^{90} Y \text { and its mean } \\
\text { square deviation (in } \\
\% \text { ) }\end{array}\end{array}$ \\
\hline${ }^{235} \mathrm{U}$ fission rate \\
\hline${ }^{239} \mathrm{Pu}$ fission rate \\
\hline $\begin{array}{c}4.92 \cdot 10^{14}+/-0.82 \\
4.92 \cdot 10^{14}+/-7.62\end{array}$ \\
\hline $\begin{array}{c}\text { Total fission rates of } \\
{ }^{235} \mathrm{U} \text { and }{ }^{239} \mathrm{Pu}\end{array}$ \\
$4.92 \cdot 10^{14}+/-7.66$
\end{tabular}

\begin{tabular}{|c|c|}
\hline Run parameter & $\begin{array}{l}\text { Nuclear concentration } \\
\text { of }{ }^{90} \mathrm{Y} \text { and its variation } \\
\text { (in \%) }\end{array}$ \\
\hline${ }^{235} \mathrm{U}$ fission rate & $4.92 \cdot 10^{14}+/-0.81$ \\
\hline${ }^{239} \mathrm{Pu}$ fission rate & $4.92 \cdot 10^{14}+/-7.63$ \\
\hline $\begin{array}{l}\text { Total fission rates of } \\
{ }^{235} \mathrm{U} \text { and }{ }^{239} \mathrm{Pu}\end{array}$ & $4.92 \cdot 10^{14}+/-7.67$ \\
\hline
\end{tabular}

TABLE 3: Comparison of statistical and variation approach for assessment of uncertainties of nuclear concentration of ${ }^{238} \mathrm{Pu}$.

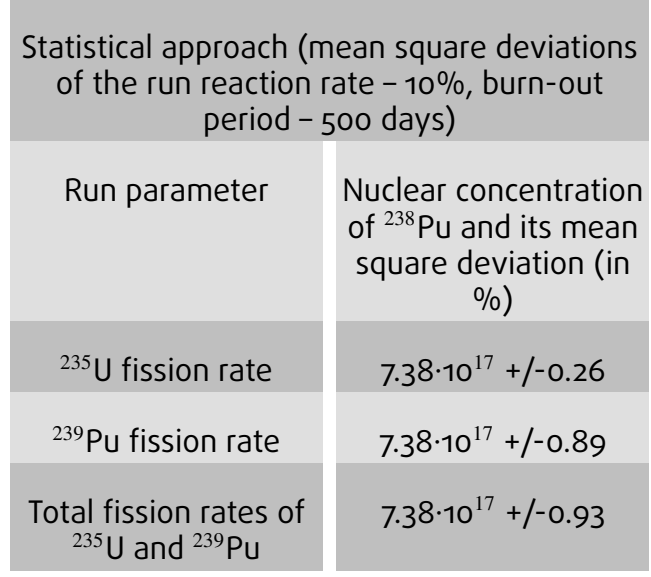

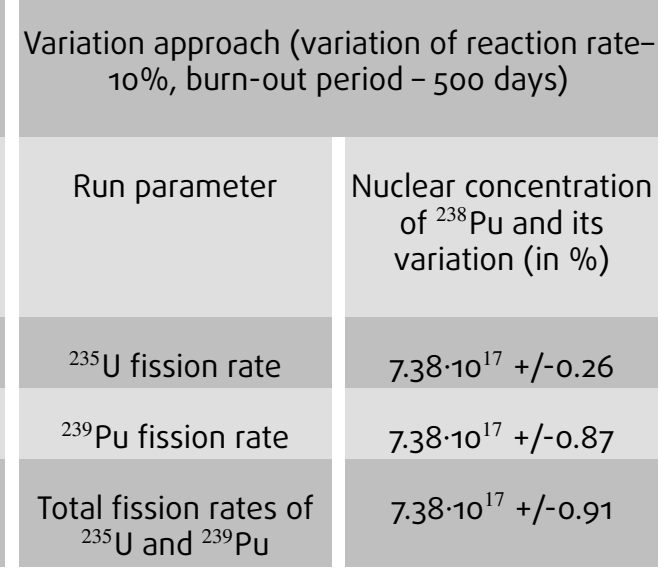

\section{Conclusions}

The results of the conducted studies show that the mean square deviations of nuclear concentration obtained using statistical approach coincide with the variations of nuclear concentrations obtained in the variation approach. Thus, for assessment of impact of uncertainties of reaction rates on the nuclear concentration obtained from 
the solution of the problem of burn-up it is possible to use less labor-consuming variation approach for assessment of their uncertainties.

\section{References}

[1] A. Gandini, M. Salvatores, L. Tondinelli. New Developments in Generalized Perturbation Methods in the Nuclide Fields. NSE, 1977, vol. 62, p. 339-344.

[2] L.N Usachev, Yu.G. Bobkov, A.S. Krivtsov. Perturbation Theory and Analysis in Fission Products Kinetics. Proc. of the Int. Conf. "Nuclear Cross-Section for Technology", Krokswill, USA, 1979. p.4.

[3] N. Garcia-Herranz, O. Cabellos, J. Sanz et. al. Propagation of Statistical and Nuclear Data Uncertainties in Monte Carlo Burn-up Calculations. Annals of Nuclear Energy, 2008 , vol. 35, issue 4, p.p. 714-730

[4] I. Kodeli. Experience with Covariance Matrix Processing. Workshop on NJOY-2005. NEA/OECD.

[5] G. Korn. T. Korn, Mathematics handbook for scientists and engineers. Definitions, theorems, formulas. - Nauka, M., 1973, 832 p.

[6] VisualBurnOut program. Authors: V.V. Kolesov, D.V. Khitrik, D.A. Kamaev. Registration No. 2009617021 dated 23.12.2009 in Computer program register.

[7] Briesmeister, J.F. (Ed.), 2000. MCNP - A General Monte Carlo N-Particle Transport Code, Version 4C. LA-13709-M, Los Alamos National Laboratory.

[8] J. Stepanek, P. Vontobel. EIR Results for the HCLWR NEACRP Burn-out Benchmark Obtained Using EIR Version of DANDE System and JEF Library. Отчет NEACRP-A-851 (1987). 